\title{
Lung Ultrasound using a Handheld Device to Diagnose COVID-19 in the Emergency Department
}

\author{
Abdulrahman M. Alfuraih ${ }^{1 *}$, (I), Abdussalam A. Alshehri ${ }^{2}$, Hani M. Alshehri ${ }^{2}$, Sami A. Alamri ${ }^{2}$, \\ Tariq S. Aleyyed ${ }^{2}$, Khalid G. Alaufi ${ }^{2}$, Mohammed J. Alsaadi ${ }^{1}$ \\ ${ }^{1}$ Radiology and Medical Imaging Department, College of Applied Medical Sciences, Prince Sattam bin Abdulaziz University, Kharj, Saudi Arabia \\ ${ }^{2}$ Emergency Department, Prince Mohammed Bin Abdulaziz Hospital, Riyadh, Saudi Arabia
}

ARTICLE INF O
Article History
Received 09 August 2021
Accepted 12 August 2021
Keywords
COVID-19
SARS-CoV-2
point-of-care ultrasound
diagnostic test
sensitivity
specificity

\section{ARTICLE INFO}

Received 09 August 2021

Keywords

COVID-19

point-of-care ultrasound

specificity

\begin{abstract}
The Coronavirus Disease 2019 (COVID-19) pandemic has critically struck healthcare systems and burdened emergency services. To date, there is no accurate and rapid point-of-care diagnostic test. This study aimed to investigate Lung Ultrasound (LUS) against Reverse Transcription-Polymerase Chain Reaction (RT-PCR) test in suspected COVID-19 patients presenting to the emergency department. In 20 eligible patients (mean age \pm standard deviation, $49 \pm 15$ years), 12 had a positive RT-PCR test and undergone an LUS examination over 12 lung zones using a handheld ultrasound device. Each zone was semiquantitatively scored according to the Lung Ultrasound Scoring System (LUSS) from 0 to 3 based on the severity of findings (pleural line irregularity, B-lines, consolidations) and documented the presence of light beam artifacts. A second blinded reader scored the images to investigate interreader reproducibility. The LUSS score had a modest diagnostic performance at $66.6 \%$ [95\% Confidence Interval (CI), 34.9-90.0\%] sensitivity and 75.0\% (95\% CI, 34.9-96.8\%) specificity. The light beam artifact was more prevalent and sensitive to COVID-19 patients with $81.8 \%$ (95\% CI, 48.2-97.7\%) sensitivity and 75.0\% (95\% CI, 34.9-96.8\%) specificity. LUS had an almost perfect interreader reproducibility for LUSS (Kendall's $W=0.961 ; 95 \%$ CI, 0.894-0.985) and light beam artifact (Cohen's $\kappa=0.890 ; 95 \%$ CI, 0.683-1.00). Overall, LUS using handheld devices can offer a safe, reproducible, rapid, and feasible first-line tool for detecting COVID-19 patients in emergency departments. The light beam artifact was more sensitive and specific to COVID-19 patients and can be useful for effectively triaging suspected cases.
\end{abstract}

(C) 2021 Dr. Sulaiman Al Habib Medical Group. Publishing services by Atlantis Press International B.V. This is an open access article distributed under the CC BY-NC 4.0 license (http://creativecommons.org/licenses/by-nc/4.0/).

\section{INTRODUCTION}

Numerous healthcare systems are facing the daily challenges of managing the Coronavirus Disease 2019 (COVID-19) pandemic. The virus spread worldwide, and close to 2.5 million lives were lost as of early 2021. Recent reports suggest that the actual COVID-19 attributable mortality is reflected by more than 20.5 million years of life lost globally [1]. Although most cases with COVID-19 develop a mild illness, approximately $14 \%$ develop severe disease requiring hospital admission, and 5\% require intensive care unit support [2]. Despite the recent breakthroughs and distribution of vaccines, a recent survey showed that $89 \%$ of scientists believe the virus is "here to stay" for years because it has spread worldwide where global eradication is unlikely [3].

Emergency physicians are confronted with large cohorts of suspected cases amidst this pandemic. They are searching for feasible and accurate diagnostic instruments to identify suspected cases. The Reverse Transcription-Polymerase Chain Reaction (RT-PCR)

Corresponding author. Email: a.alfuraih@psau.edu.sa

Peer review under responsibility of the Dr. Sulaiman Al Habib Medical Group Data availability statement: The data that support the findings of this study are owned by Dr Sulaiman Al Habib Medical Services Group Company (HMG). It can be made available from the corresponding author [AMA], upon reasonable request and conditional to HMG approval. test through a nasopharyngeal or oropharyngeal swab has been widely recognized as the reference test for diagnosing suspected cases. However, the test requires specialized laboratories; a luxury numerous countries are lacking [4]. It is also subject to delays, where results might take up to $8 \mathrm{~h}$ to be available. Additional critical disadvantages include the problematic false negative rates as well as logistic challenges [5]. Alternative radiologic tests such as chest X-ray and Computed Tomography (CT) have been used in adjunct to RT-PCR. These tests carry further disadvantages related to the capacity and use of ionizing radiation besides questionable diagnostic accuracy. The American College of Radiology (ACR) discourages such tests as the first line of investigation or screening for suspected COVID-19 [6]. They recommend a sparing use of CT to hospitalized or critical cases where they are clinically indicated.

In contrast, point-of-care Lung Ultrasound (LUS) is widely adopted in emergency departments. It can be safely performed at the bedside by the attending physician without patient transportation and risks of exposure to additional personnel. This real-time, noninvasive, and radiation-free tool is routinely used for detecting and monitoring pulmonary abnormalities. LUS has a high sensitivity (97\%) and specificity (95\%) for detecting pulmonary edema and more than $93 \%$ accuracy for pleural effusions [7].

Coronavirus disease 2019 predilection to the peripheral distribution of pathology and pleural involvement proposes a promising 
role for LUS. Indeed, there is a growing research activity for LUS in COVID-19. Multiple studies have attempted to evaluate the role of LUS in diagnosis and management in emergency departments where it is hypothesized to be useful for triaging suspected COVID-19 patients by directing suspected cases to the isolation area or discharging them. Early signs of LUS in COVID-19 showed a presence of B-lines, consolidations, pleural thickening, and lack of pleural effusions in symptomatic cases [8,9]. Although the early findings of pulmonary signs are promising, most of the studies were of retrospective design and lacked a control group by investigating confirmed COVID-19 patients. There is also considerable disagreement in the interpretation of LUS findings. Manivel et al. [10] have recently proposed the Lung Ultrasound Scoring System (LUSS) as a semiquantitative criterion for diagnosing COVID-19 in the emergency department. However, its diagnostic performance and reproducibility have not been analyzed. Volpicelli et al. [11] recently reported a typical artifact coined as the "light beam" artifact that manifests as a broad band-shaped vertical artifact arising from a normal pleural line. Case studies observed this pattern invariably in most COVID-19-related pneumonia [12]. Others speculated that this artifact is specific to early COVID-19 because of the patchy pattern of the ground-glass opacity lesions present on CT [13]. Systematic evaluation of this artifact in more than case studies is lacking. Moreover, handheld devices' performance in diagnosing COVID-19 cases is unknown despite their recommended use in emergency departments during the pandemic [14].

This study aims to investigate the diagnostic performance of LUS patterns in detecting COVID-19 using a handheld ultrasound device compared to RT-PCR in symptomatic patients presenting to the emergency department. Another study objective is to investigate the diagnostic accuracy of the recently reported light beam artifact.

\section{MATERIALS AND METHODS}

\subsection{Study Design and Patient Population}

The study was designed as a prospective case-control study at Prince Mohammed bin Abdulaziz Hospital in Riyadh, Saudi Arabia, from August 2020 to January 2021. Ethical approval was obtained (KACS registration number: H-01-R-012; NIH registration number: IRB00010471) from the institutional review board at King Fahad Medical City, the external governing ethical site. All patients provided written informed consent.

Eligible suspected and confirmed COVID-19 patients presenting to the emergency department were consecutively invited to participate using convenient sampling. The inclusion criteria were: (1) adult (>18 years); and (2) suspected COVID-19 presenting with at least one of the following symptoms: $1=$ fever $\left(>38^{\circ} \mathrm{C}\right), 2=$ cough, $3=$ shortness of breath (Medical Research Council dyspnea score $\geq 4$ ), and 4 = desaturation $\left(\mathrm{O}_{2} \leq 94\right.$ in room air). Patients unable to provide consent by themselves or an authorized relative were excluded. An RT-PCR test was performed for all patients and considered the reference test to confirm the diagnosis of COVID-19. The latest swab results were collected, including any repeat swabs. A positive result categorized the patient as a positive COVID-19 case. Blood gas analysis through venous samples were performed including partial pressure of $\mathrm{O}_{2}\left(\mathrm{pO}_{2}\right)$, partial pressure of $\mathrm{CO}_{2}\left(\mathrm{pCO}_{2}\right)$, hemoglobin oxygen saturation $\left(\mathrm{SO}_{2}\right)$, bicarbonate $\left(\mathrm{HCO}_{3}\right)$, and $\mathrm{pH}$ levels.

\subsection{Ultrasound Imaging and Scoring}

The patients were scanned using the Butterfly iQ (Butterfly Network Inc., Guilford, CT, USA) with a probe size of $185 \times 56 \times 35 \mathrm{~mm}^{3}$ equipped with 9000 micromachined sensors. The lung preset with a default depth of $13 \mathrm{~cm}$ operating at frequency $1-5 \mathrm{MHz}$ was used across all examinations. We followed the 12-zone protocol (Figure 1), which divides each lung into six zones (superior/inferior, anterior/ lateral/posterior) [15]. The probe was placed longitudinally in each zone and a still B-mode image was recorded.

Scanning was performed by the attending Emergency Room (ER) physicians, all of whom are board-accredited with prior knowledge and training in LUS. To standardize image interpretation, they were provided with case examples demonstrating the different ultrasound scores $[10,16,17]$. The LUSS from the COVID-19 Lung Ultrasound in Emergency (CLUE) protocol was utilized to score the images $[10,16,17]$. Each zone was scored from 0 to 3 . The definition for each score is as follows: score $0=$ smooth pleural line, A-lines, maximum of 2 B-lines; score 1 = irregular or thickened pleura, $>2$ B-lines; score 2 = subpleural consolidation $(<1 \mathrm{~cm}$ thickness), confluent B-lines; and score $3=$ large consolidation $(>1 \mathrm{~cm})$, air bronchograms. According to the LUSS-CLUE protocol, the patients were classified based on the total score (0-36) as normal (score 0), mild (score 1-5), moderate (score 6-15), and severe (>15) [10]. Moreover, the patients were dichotomized, based on a previously reported threshold, as positive if they had a score $>12$ [18]. The images were also retrospectively evaluated for the presence of the light beam artifact, which is characterized as a thick and strong hyperechoic shining band of confluent B-lines artifact arising from a relatively smooth pleura and surrounded by spared areas of A-lines [11,13]. The physician had an aid to record the score for each zone while scanning.

To test for LUSS interreader reproducibility, an external reader with 10 years of experience in ultrasonography-who was blinded to the original scores and clinical information including RT-PCR results-retrospectively recorded the images. Finally, the examination duration was retrospectively calculated to evaluate the feasibility by calculating the time difference between the first and last images.

\subsection{Statistical Analysis}

Descriptive and inferential statistics were done using SPSS version 27 (IBM Corp., Armonk, NY, USA). Fisher's exact test was used

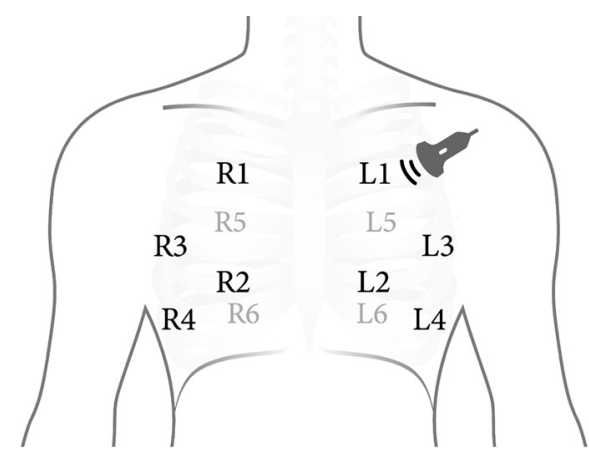

Figure 1 The 12 lung ultrasound zones protocol. Zones 1 and 2 represent the anterior lung; zones 3 and 4, lateral lung; and zones 5 and 6 , posterior lung zones. 
to compare the controls against COVID-19 patients for dichotomous categorical variables. To compare the two groups in terms of continuous variables, an independent samples $t$-test was used or the alternative nonparametric Mann-Whitney $U$-test when the data were not normally distributed. The Receiver Operating Characteristic (ROC) curve was used to represent the overall diagnostic performance of the LUSS by computing the area under ROC curve. The 95\% Confidence Intervals (CIs) were provided and computed using the exact binomial method. A $p$-value $<0.05$ was regarded as statistically significant. No missing or indeterminate data were encountered.

The interrater reproducibility of the ordinal LUSS of each image was analyzed using Kendall's coefficient of concordance $(W)$, which provides an agreement coefficient $(W)$ between 0 and 1 , where 1 indicates that all readers ranked the images perfectly in agreement with each other [19]. In addition, the interrater reproducibility on the total LUSS score for each patient was analyzed using Intraclass Correlation Coefficients (ICCs). The interreader reproducibility in detecting the light beam artifact was tested using Cohen's kappa $(\kappa)$. The reproducibility results were interpreted as follows: $0.00-0.20=$ poor agreement; $0.21-0.40=$ fair agreement; $0.41-0.60=$ moderate agreement; $0.61-0.80=$ substantial agreement; and $>0.80=$ almost perfect agreement [20].

\section{RESULTS}

\subsection{Patient Characteristics}

A total of 20 patients participated in this study. The mean age \pm standard deviation was $49 \pm 15$ (range 20-71) years, and three of the patients were female. A total of eight patients (40\%) who had a negative RT-PCR test result were considered controls. The demographic and clinical characteristics of COVID-19 patients and controls are listed in Table 1. The complete patient characteristics including blood gas analysis results are included in Supplementary Table S1. Those in the control group were on average younger than those in the COVID-19 group by approximately 14 years ( $p=0.044)$. The most frequent symptoms were cough $(80 \%)$ and shortness of breath (75\%) followed by tiredness/lethargy (50\%), sore throat $(45 \%)$, and chest pain $(45 \%)$. Six patients $(30 \%)$ presented with fever $\left(>38.0^{\circ} \mathrm{C}\right)$.

\subsection{Lung Ultrasound}

All participants had a complete LUS scan. The median scanning duration was 10.5 minutes and ranged between 5 and $16 \mathrm{~min}$. Pleural effusions were detected in one COVID-19 patient and in one control. The LUSS was almost double in COVID-19 patients, with a median score of 17 compared to 9.5 in controls (Table 1 ). However, this difference was not statistically significant $(p=0.17)$ because of the wide variation in the readings (interquartile range $=13$ ). Indeed, the ROC curve (95\% CI) for LUSS was modest at 0.693 (95\% CI, 0.449-0.936; $p=0.153$ ) indicating poor discrimination between the patients and controls (Figure 2). When categorizing patients $>12$ points as positive [18], the diagnostic performance was also modest at $66.6 \%$ (95\% CI, 34.9-90.0\%) sensitivity and 75.0\% (95\% CI, 34.9-96.8\%) specificity. Higher LUSS scores of approximately $30 \%$ were noted
Table 1 Demographic, clinical and ultrasound findings of the COVID-19 patients and controls

\begin{tabular}{|c|c|c|c|}
\hline Characteristic & $\begin{array}{l}\text { COVID-19 } \\
\quad(n=12)\end{array}$ & $\begin{array}{c}\text { Controls } \\
(n=8)\end{array}$ & $\begin{array}{c}P \text { for } \\
\text { difference }\end{array}$ \\
\hline Age $(\text { years })^{\mathrm{a}}$ & $54.7(12.6)$ & $40.4(14.6)$ & 0.044 \\
\hline Sex (M:F) & $9: 3$ & $8: 0$ & - \\
\hline Oxygen saturation in room air & $86.5(8.7 \%)$ & $90.6(9.9 \%)$ & 0.36 \\
\hline Required oxygen therapy & 9 & 4 & 0.25 \\
\hline Never smoked & $11(91 \%)$ & $7(87 \%)$ & 0.65 \\
\hline \multicolumn{4}{|l|}{ Comorbidities } \\
\hline Heart disease & 2 & 3 & - \\
\hline Hypertension & 4 & 1 & - \\
\hline Diabetes & 5 & 2 & - \\
\hline Asthma & 1 & 1 & - \\
\hline COPD & 1 & 0 & - \\
\hline \multicolumn{4}{|l|}{ Symptoms } \\
\hline Fever $\left(>38.0^{\circ} \mathrm{C}\right)$ & 5 & 1 & - \\
\hline Cough & 10 & 5 & - \\
\hline Dyspnea & 9 & 5 & - \\
\hline Sore throat & 6 & 3 & - \\
\hline Chest pain or pressure & 6 & 3 & - \\
\hline Loss of taste and/or smell & 3 & 1 & - \\
\hline Joint pain & 5 & 3 & - \\
\hline Lethargy/tiredness & 8 & 2 & - \\
\hline Aches and pains & 3 & 1 & - \\
\hline Headache & 6 & 2 & - \\
\hline Lung ultrasound score ${ }^{\mathrm{b}}$ & $17(18)$ & $9.5(12)$ & 0.17 \\
\hline Presence of light beam artifact & $9(75 \%)$ & $2(25 \%)$ & 0.024 \\
\hline
\end{tabular}

a Reported as mean (standard deviation). ${ }^{b}$ Reported as median (interquartile range). COPD, chronic obstructive pulmonary disease.

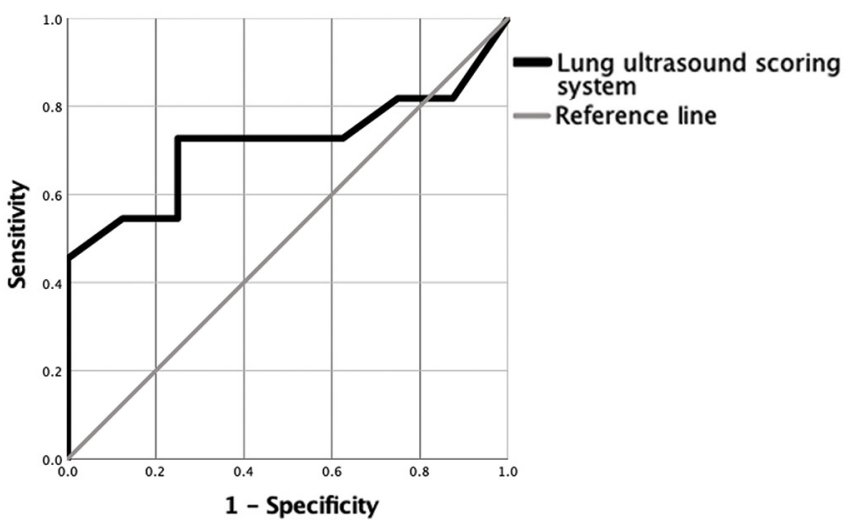

Figure 2 Receiver operating characteristic curve for the diagnostic performance of the Lung Ultrasound Scoring System (LUSS).

in the inferior lung zones. As for the LUSS classifications, the Chisquare test of homogeneity showed that the proportions were not significantly different between the two groups $(p=0.11)$. Nevertheless, there was a particularly larger number of COVID-19 patients than controls in the severe classification (Figure 3).

The light beam artifact was detected in 11 patients, nine of whom had COVID-19 (Figure 4). The difference in proportions was statistically significant $(p=0.024)$. The results diagnostically achieved 81.8\% (95\% CI, 48.2-97.7\%) sensitivity, 75.0\% (95\% CI, 34.9$96.8 \%$ ) specificity, $82 \%$ (95\% CI, 56.7-93.9\%) positive predictive value, $75.0 \%$ (95\% CI, 44.6-91.8\%) negative predictive value, and an overall accuracy of $78.9 \%$ (95\% CI, 54.4-93.9\%). 


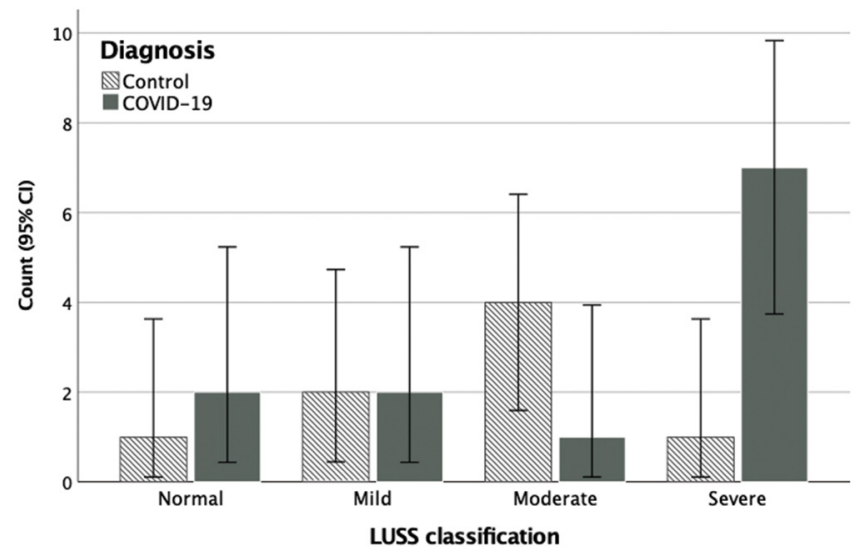

Figure 3 Frequency distribution of the lung ultrasound scoring system (LUSS) classifications for COVID-19 patients and controls.

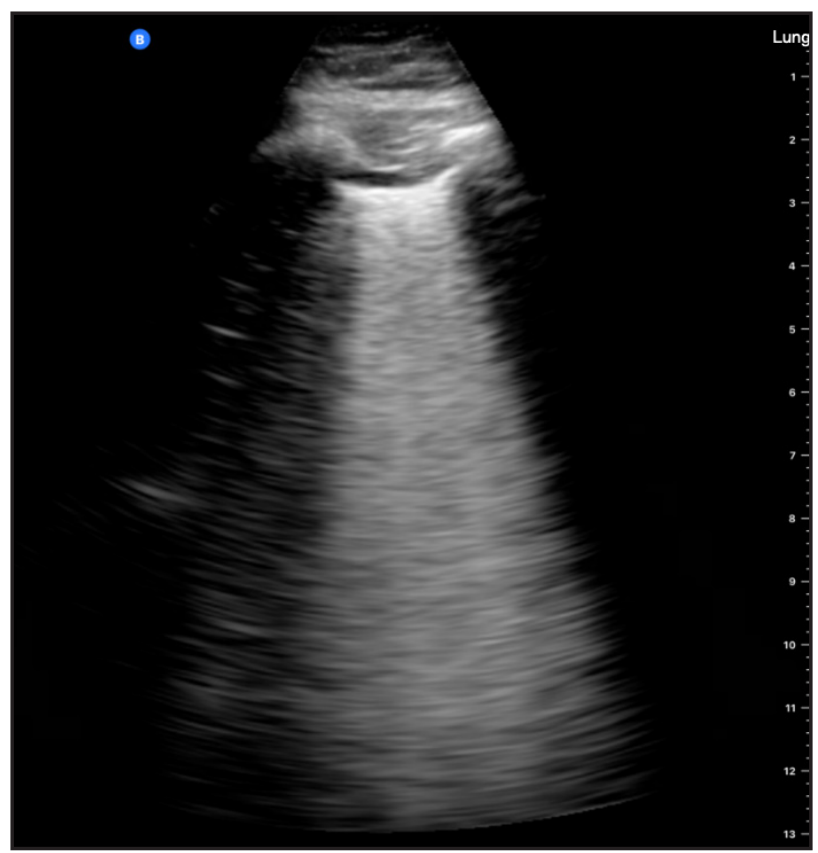

Figure 4 Light beam artifact detected in a 51-year-old male with COVID-19 (positive RT-PCR).

Kendall's $W$ was used to determine the interrater reproducibility of LUSS for each image. The result indicated statistically significant high agreement in their ratings, $W=0.832, p<0.001$. The single measures ICC for interrater reproducibility of the total LUSS score was 0.961 (95\% CI, 0.894-0.985), demonstrating almost perfect agreement. Cohen's $\kappa$ was run to determine the agreement in detecting the light beam artifact. The results also indicated an almost perfect agreement $(\kappa=0.890$; 95\% CI, 0.683-1.00; $p<0.001)$. No adverse events were noted from performing the LUS or RT-PCR tests.

\section{DISCUSSION}

To the best of our knowledge, no previous studies have investigated the diagnostic performance of LUS using handheld devices in the emergency department. This study is also the first to report the performance of the previously proposed LUSS in COVID-19. Overall, our results demonstrated a modest diagnostic performance for the LUSS. This suggests that patients with or without COVID-19 may not be accurately categorized on the basis of the total LUS score. However, the light beam artifact was a more characteristic pattern and resulted in superior diagnostic performance.

The LUSS is a promising newly proposed semiquantitative scoring system in the context of COVID-19 diagnosis. Despite a larger average score in COVID-19, our data showed that the total LUSS score was not significantly different between COVID-19 patients and controls when using RT-PCR as the reference test. The modest diagnostic performance we observed corresponds with what Şan et al. [21] had reported (62.5\% sensitivity and specificity). Raiteri et al. [22] used the relatively similar intensive care unit LUS scoring system and reported low sensitivity (66.7\%) but slightly better specificity (85.6\%). Meanwhile, Bosso et al. [18] found a marginally higher sensitivity (73\%) and specificity (89\%). By contrast, other groups [23-27] used alternative scoring methodologies and reported high sensitivities ranging from $90 \%$ to $100 \%$. The specificity was a consistent trade-off in most studies [22,24,27,28]. Our data failed to reach these high diagnostic performances using the different LUSS thresholds we implemented. The modest specificity stems from the fact that popular LUS signs, such as B-lines, are not pathognomonic for COVID-19 [29]. It should be stressed that the described high performance of common signs might be confounded by the high prevalence of such cases presenting to the emergency departments amidst the pandemic. Hence, specificity and sensitivity can be improved when LUS is integrated with clinical assessment [30,31].

The diagnostic performance discrepancy noticeable in the LUS in COVID-19 literature can be explained by the varying definitions of LUS outcome measure (i.e., how to interpret the images). Some studies have merely used any presence of B-lines or consolidations indicative of COVID-19 [21,25,27,32]. Others developed an arbitrary LUS scoring system based on a 5-point Likert scale [24] or a 6-point system [28]. There is an apparent disagreement on the scoring of small consolidations, where sometimes they are scored as 1 whereas in other studies they are scored as 2 [22]. In contrast, other researchers deemed consolidations as signs against COVID-19 pneumonitis [23]. The promising standardized LUSS may, unfortunately, not be the called for solution to standardize LUS image interpretation based on our findings. Nevertheless, further research on a larger sample size can elucidate the observed high scores in the COVID-19 group. Recently, Volpicelli et al. [33] developed a mutually exclusive classification system that categorizes the findings as low, intermediate, high, or alternative probability for COVID-19. Their system used a qualitative approach for classifying the patients, which yielded excellent accuracy.

The light beam artifact was originally hypothesized to reflect a patchy localization of COVID-19 pneumonia. It has been specifically described as "a shining band-form artifact spreading down from a large portion of a regular pleural line, often appearing and disappearing with an on-off effect in the context of a normal A-line lung pattern visible in the background" [11]. It is thought to represent an early sign for viral spread in the peripheral lung corresponding with the ground-glass opacifications detected on CT. However, few researchers argue the specificity of the light beam artifact to COVID-19, claiming that it is simply "a patchy area of 
white lung" [34]. In contrast, our data showed that it had a high diagnostic yield as a typical LUS feature of COVID-19 and can be reliably detected using a handheld ultrasound device. Indeed, our results correspond to Volpicelli et al.s [33] findings that suggested its value as an independent predictor of RT-PCR positivity.

As with all ultrasound artifacts, the light beam artifact can be influenced by the probe orientation, machine settings, and probe type. We recommend using a convex profile probe operating at low frequency $(<3 \mathrm{MHz})$ with the focus positioned at the pleural line for accurate interpretation. Notwithstanding, our results support the role of the light beam artifact as a more sensitive and specific pattern compared to conventional patterns in LUS. This positive finding agrees with a few published case reports and observations $[11,13,35]$. Our study is the first to systematically analyze this artifact in an observational study on COVID-19. An international multicenter research is currently ongoing to investigate the specificity to particular COVID-19 phenotypes [11,35].

Few studies have evaluated the interreader agreement of LUS in COVID-19. A recent study by Kumar et al. [36] investigated the reproducibility of LUS qualitative findings. They reported a substantial interreader reproducibility of 0.79 (95\% CI, 0.72-0.87) kappa value for normal LUS, single, and multiple B-lines but moderate $(\kappa=0.57 ; 95 \% \mathrm{CI}, 0.50-0.64)$ and fair $(\kappa=0.23 ; 95 \%$ CI, $0.15-0.30)$ reproducibility for consolidations and pleural thickening, respectively. Other groups used the relatively similar intensive care unit LUS scoring system and reported a substantial interreader reproducibility $(\kappa=0.79)$ [22]. When investigating the reproducibility of the semiquantitative LUSS, this study demonstrated promising agreement between readers. This finding was also true for the characteristic light beam artifact. Despite the promising findings of such subjective LUS findings, the introduction of an automated computer-aided quantitative scoring technique might offer an objective and operator-independent assessment [37].

In terms of feasibility, Wolfshohl et al. [38] conducted a survey on emergency physicians which suggested the majority were not prepared to perform LUS in emergency settings because of lack of targeted training despite their awareness of its superior diagnostic performance. In contrast, Narinx et al. [26] reported a more positive experience in their study, which agrees with our experience, in which LUS was a useful, rapid, and safe tool in the ER. In our study, all physicians were trained and reported no scanning predicaments. The reported average scanning time of 10 min supports the feasibility of LUS in the emergency setting for COVID-19 cases.

The majority of published studies used cart-based ultrasound systems. Our study is the first to investigate the handheld Butterfly IQ device in an emergency setting. A single previous study retrospectively investigated this device in comparison to a high-end ultrasound system on hospitalized COVID-19 patients [39]. Bennett et al. [39] reported an excellent concordance coefficient (95\% CI) of 0.989 (0.978-0.994). Such handheld devices are relatively cheaper and demonstrated excellent reproducibility. The most valuable feature is the small size factor, which suggests lower infection risks because of the relative ease of disinfection. They can also be encased in a cover and are not equipped with a cooling fan. Indeed, infection control is paramount in this pandemic, and several scientific societies have endorsed the use of handheld ultrasound devices for COVID-19 [14]. However, handheld devices offer limited to no adjustments to the technical sonographic settings such as focusing and frequency manipulation. They also come with a few trade-offs, such as lower spatial resolution and smaller screen size. Our reported lower diagnostic performance for the overall LUSS score might be confounded by the imaging ability of the handheld device. This explanation can be supported by the reported substantial interrater reproducibility when interpreting the images.

We support the use of LUS as a bedside assessment tool for suspected COVID-19 patients in emergency settings because of several advantages. It can be useful for prompt isolation of patients with suggestive COVID-19 findings, such as the light beam pattern and high LUSS scores. LUS can be carried out using rapid and simplified protocols. Dacrema et al. [40] adopted a simplified LUS protocol for COVID-19 diagnosis focusing on six posterior zones only, which yielded excellent sensitivity. Furthermore, we support the sole use of LUS and sparing chest X-ray and CT for selected and critical cases, in agreement with the Saudi COVID-19 safety guide for healthcare workers, the Multinational Consensus Statement from the Fleischner Society, and ACR $[6,41]$.

This study had several limitations. The sample size was small for several reasons, including the steep decline in COVID-19 cases during the recruitment phase. The number of rejections to participate was not recorded. However, it was deemed relatively high (approximately $4: 1$ ), especially by female patients because of the examination's revealing nature. The study did not correlate LUS with variables for arterial blood gas analysis or CT because of the data's unavailability. The positive and negative predictive values we reported on the light beam artifact should be interpreted cautiously as they are highly dependent on the disease's prevalence. They will unlikely represent the true predictive values in other communities or globally because of the fluctuating prevalence of COVID-19. More longitudinal research is warranted for investigating the prognostic role of LUS concerning mortality, ICU admission, and intubation as well as its value in patient management. This is merited with reports suggesting LUS can lead to overdiagnosis leading to higher hospital admissions, which may further overwhelm healthcare systems [42].

\section{CONCLUSION}

In conclusion, semiquantitative scores of LUS findings using the LUSS were relatively higher in COVID-19 patients than controls but had limited diagnostic performance for identifying suspected cases compared to RT-PCR as a reference test. In contrast, a recently recognized qualitative sign called the light beam artifact had superior sensitivity and specificity. Overall, the results suggest that LUS can be feasibly and reliably implemented in the emergency department while waiting for the RT-PCR confirmatory results. Owing to modest specificity, suggestive LUS outcomes with a positive light beam artifact should be confirmed with the RT-PCR test. Future research should investigate the temporal changes of LUS findings and study their prognostic value.

\section{CONFLICTS OF INTEREST}

The authors declare they have no conflicts of interest. 


\section{AUTHORS' CONTRIBUTION}

AMA and MJA contributed in study conceptualization. AMA, AAA, HMA, SAA, TSA and KGA contributed in study design. AAA, HMA and SAA contributed in data collection. AMA and MJA contributed in data interpretation and analysis. AMA and MJA contributed in manuscript writing and preparation. All authors reviewed the final draft.

\section{FUNDING}

This research has been funded under Dr Sulaiman Al Habib Medical Journal grant to support COVID-19 research. The funding body had no role or influence in the data interpretation or conclusions.

\section{ACKNOWLEDGMENTS}

The research team is grateful for Al Habib Medical Group for funding this project in support of local COVID-19 research. The authors are thankful to the emergency department residents (Dr. Abdullah Alshehri, Dr. Nader Bokhari, Dr. Mansour Aldhafeeri, Dr. Malek Alaaullah) and supporting staff as well as the research participants for their contribution to the research.

\section{SUPPLEMENTARY MATERIAL}

Supplementary data related to this article can be found at https:// doi.org/10.2991/dsahmj.k.210823.001

\section{REFERENCES}

[1] Arolas HPI, Acosta E, López-Casasnovas G, Lo A, Nicodemo C, Riffe T, et al. Years of life lost to COVID-19 in 81 countries. Sci Rep 2021;11;3504.

[2] World Health Organization (WHO). Clinical management of severe acute respiratory infection (SARI) when COVID-19 disease is suspected: interim guidance, 13 March 2020. Geneva, Switzerland: WHO; 2020. Available from: https://apps.who.int/ iris/handle/10665/331446.

[3] Phillips N. The coronavirus is here to stay - here's what that means. Nature 2021;590;382-4.

[4] Giri AK, Rana DRSJB. Charting the challenges behind the testing of COVID-19 in developing countries: Nepal as a case study. Biosaf Health 2020;2;53-6.

[5] Ai T, Yang Z, Hou H, Zhan C, Chen C, Lv W, et al. Correlation of chest CT and RT-PCR testing for coronavirus disease 2019 (COVID-19) in China: a report of 1014 cases. Radiology 2020;296;E32-E40.

[6] Radiology ACo. ACR recommendations for the use of chest radiography and computed tomography (CT) for suspected COVID-19 infection 2020. Available from: https://www.acr.org/ Advocacy-and-Economics/ACR-Position-Statements/ Recommendations-for-Chest-Radiography-and-CT-forSuspected-COVID19-Infection [cited February 1, 2021].

[7] Lichtenstein D, Goldstein I, Mourgeon E, Cluzel P, Grenier P, Rouby JJ. Comparative diagnostic performances of auscultation, chest radiography, and lung ultrasonography in acute respiratory distress syndrome. Anesthesiology 2004;100;9-15.

[8] Huang Y, Wang S, Liu Y, Zhang Y, Zheng C, Zheng Y, et al. A preliminary study on the ultrasonic manifestations of peripulmonary lesions of non-critical novel coronavirus pneumonia (COVID-19). SSRN 2020.

[9] Peng QY, Wang XT, Zhang LN, Chinese Critical Care Ultrasound Study Group (CCCUSG). Findings of lung ultrasonography of novel corona virus pneumonia during the 2019-2020 epidemic. Intensive Care Med 2020;46;849-50.

[10] Manivel V, Lesnewski A, Shamim S, Carbonatto G, Govindan T. CLUE: COVID-19 lung ultrasound in emergency department. Emerg Med Australas 2020;32;694-6.

[11] Volpicelli G, Lamorte A, Villén T. What's new in lung ultrasound during the COVID-19 pandemic. Intensive Care Med $2020 ; 46 ; 1445-8$.

[12] Soldati G, Smargiassi A, Inchingolo R, Buonsenso D, Perrone T, Briganti DF, et al. On lung ultrasound patterns specificity in the management of COVID-19 patients. J Ultrasound Med 2020; 39;2283-4.

[13] Millington SJ, Koenig S, Mayo P, Volpicelli G. Lung ultrasound for patients with coronavirus disease 2019 pulmonary disease. Chest 2021;159;205-11.

[14] Kim DJ, Jelic T, Woo MY, Heslop C, Olszynski P. Just the facts: recommendations on point-of-care ultrasound use and machine infection control during the coronavirus disease 2019 pandemic. CJEM 2020;22;445-9.

[15] Rouby JJ, Arbelot C, Gao Y, Zhang M, Lv J, An Y, et al. Training for lung ultrasound score measurement in critically ill patients. Am J Respir Crit Care Med 2018;198;398-401.

[16] Soldati G, Smargiassi A, Inchingolo R, Buonsenso D, Perrone T, Briganti DF, et al. Proposal for international standardization of the use of lung ultrasound for patients with COVID-19: a simple, quantitative, reproducible method. J Ultrasound Med 2020;39;1413-19.

[17] Mongodi S, Bouhemad B, Orlando A, Stella A, Tavazzi G, Via G, et al. Modified lung ultrasound score for assessing and monitoring pulmonary aeration. Ultraschall Med 2017;38;530-7.

[18] Bosso G, Allegorico E, Pagano A, Porta G, Serra C, Minerva V, et al. Lung ultrasound as diagnostic tool for SARS-CoV-2 infection. Intern Emerg Med 2021;16;471-6.

[19] Field AP. Kendall's coefficient of concordance. In: Balakrishnan N, Colton T, Everitt B, et al., editors. Wiley StatsRef: Statistics Reference Online. Hoboken, NJ: Wiley; 2014, pp. 256-7.

[20] Landis JR, Koch GG. The measurement of observer agreement for categorical data. Biometrics 1977;33;159-74.

[21] Şan İ, Bekgöz B, Usul E, Yıldırım Ç, Gemcioğlu E, Kahraman AF, et al. Role of lung ultrasonography in the diagnosis of COVID-19 patients admitted to the emergency department. Notf Rett Med 2020;1-6.

[22] Raiteri A, Alvisi M, Serio I, Stefanini F, Tovoli F, Piscaglia F. Lung ultrasound is often, but not always, normal in healthy subjects: considerations for COVID-19 pandemic. Diagnostics 2021;11;82.

[23] Walsh P, Hankins A, Bang H. Point-of-care lung ultrasound is useful to evaluate emergency department patients for COVID-19. West J Emerg Med 2020;21;24-31.

[24] Zanforlin A, Strapazzon G, Falk M, Gallina V, Viteritti A, Valzolgher L, et al. Lung ultrasound in the emergency department for early identification of COVID-19 pneumonia. Respiration 2021;100;145-53. 
[25] Karagöz A, Sağlam C, Demirbaş HB, Korkut S, Ünlüer EE. Accuracy of bedside lung ultrasound as a rapid triage tool for suspected Covid-19 cases. Ultrasound Q 2020;36;339-44.

[26] Narinx N, Smismans A, Symons R, Frans J, Demeyere A, Gillis M. Feasibility of using point-of-care lung ultrasound for early triage of COVID-19 patients in the emergency room. Emerg Radiol 2020;27;663-70.

[27] Sorlini C, Femia M, Nattino G, Bellone P, Gesu E, Francione P, et al. The role of lung ultrasound as a frontline diagnostic tool in the era of COVID-19 outbreak. Intern Emerg Med 2021;16; $749-56$,

[28] Haak SL, Renken IJE, Jager LC, Lameijer H, van der Kolk BBYM. Diagnostic accuracy of point-of-care lung ultrasound in COVID-19. Emerg Med J 2021;38;94-9.

[29] Alfuraih AM. Point of care lung ultrasound in COVID-19: hype or hope?. BJR Open 2020;2;20200027.

[30] Pivetta E, Goffi A, Tizzani M, Locatelli SM, Porrino G, Losano I, et al. Lung ultrasonography for the diagnosis of SARS-CoV-2 pneumonia in the emergency department. Ann Emerg Med 2021;77;385-94.

[31] Allegorico E, Buonerba C, Bosso G, Pagano A, Porta G, Serra C, et al. The use of chest ultrasonography in suspected cases of COVID-19 in the emergency department. Future Sci OA 2020;7;FSO635.

[32] Schmid B, Feuerstein D, Lang CN, Fink K, Steger R, Rieder M, et al. Lung ultrasound in the emergency department - a valuable tool in the management of patients presenting with respiratory symptoms during the SARS-CoV-2 pandemic. BMC Emerg Med 2020;20;96.

[33] Volpicelli G, Gargani L, Perlini S, Spinelli S, Barbieri G, Lanotte A, et al. Lung ultrasound for the early diagnosis of
COVID-19 pneumonia: an international multicenter study. Intensive Care Med 2021;47;444-54.

[34] Taccari F, Buonsenso D. COVID-19 and lung ultrasound: reflections on the "light beam". J Ultrasound Med 2021;40;1037-8.

[35] Volpicelli G, Gargani L. Sonographic signs and patterns of COVID-19 pneumonia. Ultrasound J 2020;12;22.

[36] Kumar A, Weng Y, Graglia S, Chung S, Duanmu Y, Lalani F, et al. Interobserver agreement of lung ultrasound findings of COVID-19. J Ultrasound Med 2021.

[37] Corradi F, Via G, Forfori F, Brusasco C, Tavazzi G. Lung ultrasound and B-lines quantification inaccuracy: B sure to have the right solution. Intensive Care Med 2020;46;1081-3.

[38] Wolfshohl J, Shedd A, Chou EH, d'Etienne JP. Lung ultrasound for COVID-19 evaluation in the emergency department: is it feasible?. Ann Emerg Med 2020;76;552-3.

[39] Bennett D, De Vita E, Mezzasalma F, Lanzarone N, Cameli P, Bianchi F, et al. Portable pocket-sized ultrasound scanner for the evaluation of lung involvement in coronavirus disease 2019 patients. Ultrasound Med Biol 2021;47;19-24.

[40] Dacrema A, Silva M, Rovero L, Vertemati V, Losi G, Piepoli $\mathrm{MF}$, et al. A simple lung ultrasound protocol for the screening of COVID-19 pneumonia in the emergency department. Intern Emerg Med 2021;16;1297-305.

[41] Rubin GD, Ryerson CJ, Haramati LB, Sverzellati N, Kanne JP, Raoof S, et al. The role of chest imaging in patient management during the COVID-19 pandemic: a multinational consensus statement from the Fleischner Society. Radiology 2020;296;172-80.

[42] Gil-Rodrigo A, Llorens P, Luque-Hernández MJ, MartínezBuendía C, Ramos-Rincón JM. Lung ultrasound integration in assessment of patients with noncritical COVID-19. J Ultrasound Med 2021. 